\title{
Categorical Risk Perception Drives Variability in Antibiotic Prescribing in the Emergency Department: A Mixed Methods Observational Study
}

\author{
Eili Y. Klein, $P h D^{1,2,3}$, Elena M. Martinez, MS², Larissa May, MD', Mustapha Saheed, MD', \\ Valerie Reyna, $P h D^{5}$, and David A. Broniatowski, $P h D^{6}$
}

\begin{abstract}
'Department of Emergency Medicine, Johns Hopkins School of Medicine, Baltimore, MD, USA; ${ }^{2}$ Center for Disease Dynamics, Economics \& Policy, Washington, DC, USA; ${ }^{3}$ Department of Epidemiology, Johns Hopkins Bloomberg School of Public Health, Baltimore, MD, USA; ${ }^{4}$ Department of Emergency Medicine, University of California, Davis, Sacramento, CA, USA; ${ }^{5}$ Human Neuroscience Institute, Center for Behavioral Economics and Decision Research and Human Neuroscience Institute, Cornell University, Ithaca, NY, USA; 'Department of Engineering Management and Systems Engineering, The George Washington University, Washington, DC, USA.
\end{abstract}

BACKGROUND: Adherence to evidence-based antibiotic therapy guidelines for treatment of upper respiratory tract infections (URIs) varies widely among clinicians. Understanding this variability is key for reducing inappropriate prescribing.

OBJECTIVE: To measure how emergency department (ED) clinicians' perceptions of antibiotic prescribing risks affect their decision-making.

DESIGN: Clinician survey based on fuzzy-trace theory, a theory of medical decision-making, combined with retrospective data on prescribing outcomes for URI/ pneumonia visits in two EDs. The survey predicts the categorical meanings, or gists, that individuals derive from given information.

PARTICIPANTS: ED physicians, residents, and physician assistants (PAs) who completed surveys and treated patients with URI/pneumonia diagnoses between August 2014 and December 2015.

MAIN MEASURES: Gists derived from survey responses and their association with rates of antibiotic prescribing per visit.

KEY RESULTS: Of 4474 URI/pneumonia visits, 2874 $(64.2 \%)$ had an antibiotic prescription. However, prescribing rates varied from $7 \%$ to $91 \%$ for the 69 clinicians surveyed (65.2\% response rate). Clinicians who framed therapy-prescribing decisions as a categorical choice between continued illness and possibly beneficial treatment ("why not take a risk?" gist, which assumes antibiotic therapy is essentially harmless) had higher rates of prescribing (OR 1.28 [95\% CI, 1.06-1.54]). Greater agreement with the "antibiotics may be harmful" gist was associated with lower prescribing rates (OR 0.81 [95\% CI, 0.67-0.98]).

CONCLUSIONS: Our results indicate that clinicians who perceive prescribing as a categorical choice between patients remaining ill or possibly improving from therapy are more likely to prescribe antibiotics. However, this strategy

Electronic supplementary material The online version of this article (doi:10.1007/s11606-017-4099-6) contains supplementary material, which is available to authorized users.

Received October 17, 2016

Revised April 6, 2017

Accepted June 2, 2017

Published online June 20, 2017 assumes that antibiotics are essentially harmless. Clinicians who framed decision-making as a choice between potential harms from therapy and continued patient illness (e.g., increased appreciation of potential harms) had lower prescribing rates. These results suggest that interventions to reduce inappropriate prescribing should emphasize the non-negligible possibility of serious side effects.

KEY WORDS: fuzzy-trace theory; drivers of decision-making; nonnegligible risks of unnecessary antibiotics; gist of antibiotic therapy; antibiotic resistance.

J Gen Intern Med 32(10):1083-9

DOI: $10.1007 /$ s11606-017-4099-6

(C) Society of General Internal Medicine 2017

\section{INTRODUCTION}

Despite clear, evidence-based guidelines for antibiotic use in upper respiratory tract infections (URIs), prescribing rates and the appropriateness of prescriptions vary widely in both inpatient and outpatient settings. ${ }^{1-5}$ Quantitative research identifying drivers of inappropriate prescribing has focused mainly on patient characteristics. ${ }^{6-8}$ In both inpatient and outpatient settings, patient gender, age, insurance status, race/ethnicity, wait time, and comorbidities influence prescribing, ${ }^{6-8}$ but together explain only a limited amount of the variation in prescribing rates.

Clinician characteristics, such as specialty, training, diagnostic uncertainty, and experience, ${ }^{6,9-16}$ explain some of the variation in prescribing rates; however, most relevant studies have been qualitative. Differences in clinicians' prescribing practices have been studied more often in inpatient settings, ${ }^{11-}$ 13 yet the majority of antibiotic prescriptions occur in outpatient settings. ${ }^{17}$ Furthermore, research in outpatient settings suggests that the influence of clinician-patient interactions (e.g., satisfying patient expectations) on prescribing rates may be greater in outpatient than inpatient settings. ${ }^{6}, 9,14,15$, 18 Other acute care settings, such as emergency departments (ED), have been less studied, though clinicians in these 
settings similarly believe that patient and health system factors are primary drivers of antibiotic prescribing decisions. ${ }^{19}$

Previous research on prescribing behavior has not systematically examined the effect of clinicians' decision-making processes on antibiotic prescribing rates, limiting the ability of interventions to use behavioral strategies to reduce inappropriate antibiotic prescribing. Here, we apply empirically validated psychological theory in an attempt to understand the factors that motivate clinician prescribing for antibiotics.

\section{METHODS}

\section{Study Design and Setting}

We surveyed clinicians in two EDs, an urban academic hospital and a suburban community hospital, in Maryland. Survey results were combined with a retrospective examination of prescribing decisions for adult patients diagnosed with a URI or pneumonia in the ED between August 2014 and December 2015. Clinicians with incomplete survey responses or who had fewer than five encounters during the study period were excluded. URI encounters included patients diagnosed with acute respiratory infections (ICD-9 codes 460-465), bronchitis (ICD-9 codes 466, 490-491), cough (ICD-9 code 786.2), influenza (ICD-9 code 487), streptococcal sore throat (ICD-9 code 034 ), or other upper respiratory tract infections (ICD-9 codes 470-475). In addition, we included pneumonia (ICD-9 codes 480-486), which is often included in studies of prescribing for respiratory tract infections, ${ }^{20,}{ }^{21}$ and except for viral cases, antibiotics are almost always indicated for treatment of pneumonia, providing a useful control. Discharged patients 18 years and older who had a URI or pneumonia diagnosis were included in the analysis. Patients diagnosed with another, non-URI infection for which treatment guidelines sometimes or always indicate antibiotics were excluded. ${ }^{3}$ Appropriateness of prescription (always appropriate, may be appropriate, or not appropriate) was determined based on diagnoses as described in Fleming-Dutra et al. ${ }^{3}$ (see Online Supplementary Table 1 for appropriateness classifications by diagnosis). Patient chief complaints were captured through standard reasons for visit classification for ambulatory care at triage and categorized by body system. ${ }^{22}$

\section{Fuzzy-Trace Theory}

Any intervention that aims to address inappropriate antibiotic prescribing must consider how physicians make decisions. Our examination of physician decision-making draws on fuzzy-trace theory (FTT), a theory of medical decisionmaking involving risk. ${ }^{23}$ According to FTT, individuals form two types of mental representations: verbatim and gist. A verbatim representation of a stimulus reflects precise, detailed information (e.g., there is a $0.1 \%$ chance of an adverse reaction); in contrast, gists capture the bottom-line meaning of the stimulus (e.g., there is essentially no risk or there is some risk).
People simultaneously encode both types of representations into memory. However, they tend to rely on the simplest gists when making decisions, using more precise (e.g., verbatim) representations only when necessary. ${ }^{23-26}$ For antibiotic prescribing, FTT can be applied to understand the constructs-gists - that underlie clinicians' prescribing decisions.

Based on a review of the literature, expert physician interviews, and our prior FTT-based survey of patients, ${ }^{27}$ we developed an electronic FTT-based survey for clinicians. The simplest gist of information is often categorical, for example, the distinction between a patient staying sick or getting better. The survey included questions expressing the categorical gists pertaining to the major motivations for antibiotic prescribing. We included questions pertaining to five major gists, as well as a set of questions indexing correct knowledge (Online Supplementary Table 1). The two primary gists that we hypothesized would be most important were "why not take a risk?" (in which the downside risk of antibiotics is assumed to be negligible) and "antibiotics may be harmful" (in which the downside risk is non-negligible). Specifically, "why not take a risk?", based on FTT, hypothesizes that clinicians frame the decision process as a categorical contrast between possibly effective treatment and the certainty of the patient remaining sick (see Reyna et al. ${ }^{28}$ ). That is, clinicians frame the decision as a categorical choice between the patient staying sick for sure or possibly getting better. Providers who subscribe to this gist should treat patients with antibiotics if there is a nonnegligible possibility that their symptoms could improve with antibiotics and if antibiotics are perceived to be essentially without risk (i.e., if prescribing antibiotics is perceived as essentially harmless to the patient). ${ }^{27}$ Alternatively, "antibiotics may be harmful" measures the clinician's concern about harmful side effects and, based on the theory, should lead to a decision process in which the categorical distinction is between the status quo and potentially getting worse by taking antibiotics. We hypothesized that clinicians who frame the decision process in this manner would be less likely to prescribe.

The survey consisted of 46 Likert-scale questions, followed by two free-response questions and 15 demographic questions (Online Supplementary Table 2). Each question had a forward- and reverse-coded version (23 pairs), and questions were presented in random order to ensure that neither wording nor order biased responses. Clinicians were invited to participate by email, and two reminders were sent. Prior to answering questions, each participant read a short description of a patient with symptoms consistent with a diagnosis of a viral URI that would not, according to clinical guidelines, necessitate antibiotic treatment (see Online Supplementary Material). Responses were recorded using a seven-point Likert scale, ranging from strongly disagree $(-3)$ to strongly agree $(+3)$. Clinicians were compensated $\$ 25$ for completing the survey. The study was approved by the Johns Hopkins Institutional Review Board. 


\section{Statistical Methods}

An exploratory factor analysis (EFA) was conducted on all survey responses to identify clusters of similarly answered questions. There was no forced extraction of components. The oblimin rotation method was used with maximum likelihood extraction to generate rotated factor loadings. Factors were retained based on goodness-of-fit criteria, including root mean square error of approximation (RMSEA) and TuckerLewis Index; Heywood cases were discarded. The retained factors were then assigned to a categorical gist based on the questions associated with each factor. Scores for each clinician for each factor were estimated using multiple regression and, for robustness, by the weighted sum method. ${ }^{29}$

A multilevel mixed-effects logistic regression was used to assess factors associated with antibiotic prescribing to account for the natural clustering of encounters by clinicians. ${ }^{30} \mathrm{Ex}$ planatory variables included sociodemographic factors for patients and clinicians, characteristics of the infection and ED visit, and EFA scores. Regression models were further stratified by both clinician type and the appropriateness of the antibiotic prescription. ${ }^{3}$ For patients diagnosed with acute bronchitis (ICD-9466, 490) and either chronic bronchitis, emphysema, or chronic airway obstruction (ICD-9 491, 492, 496) (and no other diagnoses requiring antibiotics), the visit was considered an exacerbation of chronic bronchitis, which may receive antibiotics. For all other patients with multiple diagnoses, the appropriateness of antibiotic prescribing was designated to the most appropriate tier (e.g., if any diagnosis was considered "always appropriate," then prescribing for that visit was designated as "always appropriate"). All statistical analyses were conducted using Stata 14.1 (College Station, TX) and R 3.3.0 (R Core Team, Vienna, Austria, 2016).

\section{RESULTS}

\section{Clinician Survey}

A total of 138 clinicians (attending physicians, residents, physician assistants [PAs]) were invited to participate; 90

Table 1 Clinician Characteristics

\begin{tabular}{ll}
\hline \hline & $\boldsymbol{N}(\boldsymbol{\%})$ \\
\hline Total & 69 \\
Prescribing rate & $59.2 \%$ \\
$\quad$ Physician & $68.7 \%$ \\
Physician assistant & $66.6 \%$ \\
Resident & \\
Emergency department & $46(66.7 \%)$ \\
Urban, academic & $8(11.6 \%)$ \\
Community & $15(21.7 \%)$ \\
Both & $28(40.6 \%)$ \\
Provider type & $13(18.8 \%)$ \\
Physician & $28(40.6 \%)$ \\
Physician assistant & $33(47.8 \%)$ \\
Resident & $36(52.2 \%)$ \\
Gender &
\end{tabular}

completed the survey (65.2\% completion rate), of whom 69 had at least five URI or pneumonia encounters during the study period and were included in the analysis. The prescribing rate varied by clinician type, with residents having the highest rate (Table 1). Forty-eight percent were women; most $(66.7 \%)$ worked in the urban ED, while $11.6 \%$ worked at the community hospital and $21.7 \%$ saw patients at both locations. Physicians and residents constituted the majority of the sample (40.6\%, 40.6\%, respectively) and $18.8 \%$ were PAs.

Two factors were retained from the EFA. The first factor captured $20.8 \%$ of the variance in survey responses and correlated with a gist entitled "why not take a risk?". This gist suggests that physicians make a strategic choice between the patient potentially improving with antibiotic therapy or remaining sick. Employment of this strategy further implies that the potential downside risks from antibiotic therapy are seen as essentially harmless to the patient. The second factor captured $7.2 \%$ of the variance and correlated with the "antibiotics may be harmful" gist (Online Supplementary Table 3). Stronger agreement with this gist suggests greater clinician concern about harmful side effects.

\section{Prescribing Decisions}

Of the 4474 patient encounters included in this analysis, 2874 $(64.2 \%)$ were prescribed an antibiotic (Table 2). Fifty-one percent of patients' chief complaints at triage were respiratory-related, though all were diagnosed with a URI or pneumonia. The most common diagnoses were acute respiratory infections $(38.2 \%)$ and acute bronchitis (23.2\%). Antibiotic prescribing rates varied widely by clinician, both overall and by clinician type (Fig. 1). Prescribing variability was highest for diagnoses in the generalized category of acute respiratory

Table 2 Respiratory Tract Infection Patient Demographics and Clinical Characteristics

\begin{tabular}{ll}
\hline \hline & N (\%) \\
\hline Total encounters & 4474 \\
Prescribed antibiotic & $2874(64.2 \%)$ \\
Urban, academic emergency department & $3341(74.7 \%)$ \\
Infection type & \\
$\quad$ Acute respiratory infection & $1707(38.2 \%)$ \\
Bronchitis & $1036(23.2 \%)$ \\
Cough & $286(6.4 \%)$ \\
Influenza & $165(3.7 \%)$ \\
Pneumonia & $650(14.5 \%)$ \\
Streptococcal sore throat & $243(5.4 \%)$ \\
Other disease of the upper respiratory tract & $387(8.7 \%)$ \\
Seen in rapid care area & $1849(41.3 \%)$ \\
One or more comorbidities & $1202(26.9 \%)$ \\
Chief complaint respiratory & $2264(50.6 \%)$ \\
Age (years) mean (SD) & $41.8(16.8)$ \\
Race/ethnicity & \\
Black, non-Hispanic & $3087(69.0 \%)$ \\
White, non-Hispanic & $963(21.5 \%)$ \\
Other race/ethnicity & $424(9.5 \%)$ \\
Female & $2685(60.0 \%)$ \\
Fever (>100.4 ${ }^{\circ}$ F) & $366(8.2 \%)$ \\
Time of arrival & \\
7 am-3 pm & $2289(51.2 \%)$ \\
3 pm-11 pm & $1584(35.4 \%)$ \\
11 pm-7 am & $601(13.4 \%)$ \\
Influenza season & $1381(30.9 \%)$ \\
\hline & $1585(35.4 \%)$ \\
\hline &
\end{tabular}




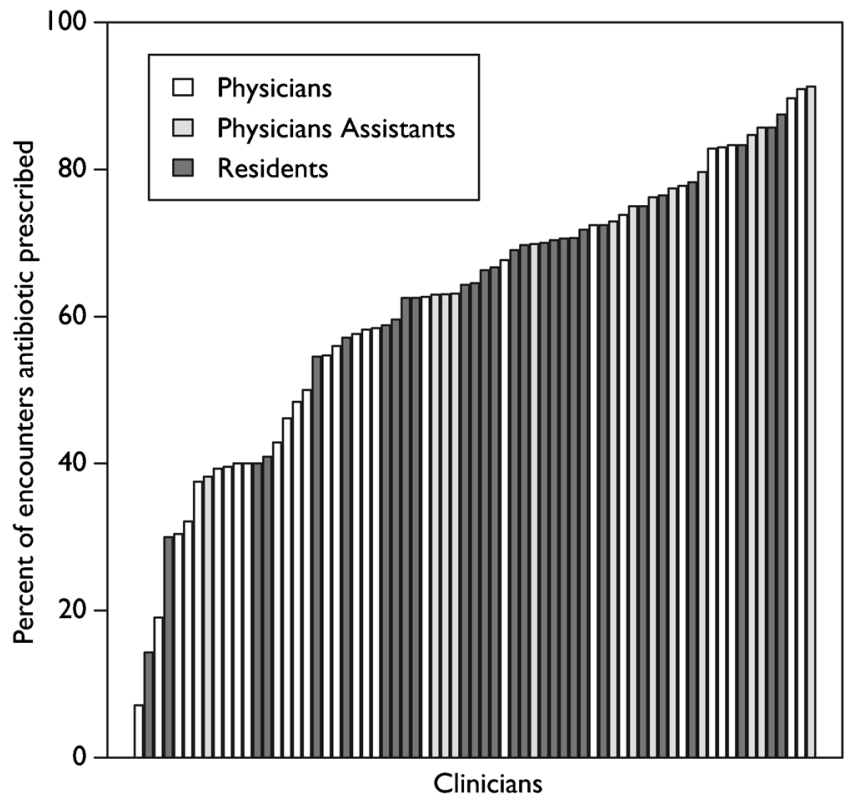

Figure 1 Variation in antibiotic prescribing rates among clinicians, separated by provider type. Each bar represents the antibiotic prescribing rate for respiratory tract infections for an individual physician, resident, or physician assistant. Physicians are represented by white bars, physician assistants by light gray bars, and residents by dark grey. Antibiotic prescribing varied widely among clinicians for all three provider types.

infections, followed by diagnoses of bronchitis, cough, and other diseases of the upper respiratory tract (Online Supplementary Figure 1). Ninety-three percent of patients with a diagnosis requiring antibiotics (bacterial pneumonia and peritonsillar abscess) received a prescription, compared to $74 \%$ of those with a diagnosis sometimes requiring antibiotics, and $50 \%$ with a diagnosis not indicating antibiotics (Online Supplementary Figure 2).

\section{Predictors of Prescribing}

The "why not take a risk?" gist was significantly associated with increased odds of antibiotic prescribing. Clinicians who agreed with this gist were more likely to prescribe (OR 1.28 [95\% CI, 1.06-1.54]) (Table 3), especially when antibiotics were not indicated (OR 1.32 [95\% CI, 1.04-1.68]). Agreement with the "antibiotics may be harmful" gist was significantly associated with decreased prescribing (OR 0.81 [95\% CI, 0.67-0.98]), especially when guidelines suggested that antibiotics may be indicated (OR 0.52 [95\% CI, 0.38-0.73]). Gist agreement with "antibiotics may be harmful" was most strongly associated with prescribing for PAs (Online Supplementary Table 4), and results were consistent when controlling for antibiotic indication (Online Supplementary Table 5).

Other factors associated with increased odds of antibiotic prescribing included patients with an initial complaint of a respiratory infection, female patients, and those with a fever. Factors associated with decreased odds of prescribing included encounters in a rapid treatment area of the ED or at the community ED. In addition, patients who arrived between
$11 \mathrm{pm}$ and 7 am were less likely to receive an antibiotic than patients who arrived during the day. The strength of these associations varied by diagnosis and clinician (Table 3 and Online Supplementary Table 4). For example, when antibiotics were not indicated, older patients, white patients, and those with long wait times were more likely to be prescribed antibiotics.

\section{DISCUSSION}

This study is the first, to our knowledge, to quantitatively link clinicians' perceptions about antibiotic prescribing to prescribing data. Our results suggest that how clinicians interpret information regarding the appropriateness of antibiotic therapy-in particular, simple categorical gist interpretations - are a significant driver of their prescribing decisions. Drawing from prior research on how individuals interpret complex medical information, ${ }^{31-33}$ we found that clinicians with higher prescribing rates appear to frame the choice as a categorical contrast between providing a possibly effective treatment and the patient remaining sick. This strategic gist representation, which we call "why not take a risk?", is distinct from a general bias towards treatment ${ }^{19}$; rather, it reflects a strategic choice based on the non-negligible possibility that the patient has a bacterial infection that may improve with antibiotic therapy. Importantly, this finding was not based on differences in beliefs regarding the effectiveness of therapy, but on a reliance on gists that frame the prescribing decision as a choice between the patient remaining sick and possibly getting better. This gist reliance assumes a broader set of motivating factors than just being safe in the face of uncertainty over infection risk. ${ }^{34}$ FTT predicts that individuals will reason primarily using categorical representations of risk, ${ }^{28}$ and based on these options will choose potential benefit over the status quo of remaining sick. FTT also predicts that clinicians who view antibiotics as essentially harmless to the patient are more likely to follow this strategy. Thus, it includes a "just to be safe" mentality, but also encompasses an absence of downside risk. Conversely, increased awareness of the potential risks of antibiotics reframes the decision-making process as one in which the clinician is choosing between the patient remaining sick and potentially getting worse due to harm from therapy. In the latter interpretation, benefits rather than risks are nil.

Individual concepts of meaning influence how clinicians interpret and respond to guidelines for prescribing, and are central to attempts to address inappropriate antibiotic prescribing. Interpreting our results within the context of FTT research on risk-taking ${ }^{28}$ suggests that educational strategies should emphasize that when antibiotics are not indicated, the potential harm to the patient from antibiotics is qualitatively or categorically worse than the status quo of the patient remaining sick, and that there is basically no benefit to the patient from inappropriate prescriptions. ${ }^{27}$ This framing of the prescribing 
Table 3 Predictors of Antibiotic Prescribing Among Emergency Department Clinicians

\begin{tabular}{|c|c|c|c|c|}
\hline \multirow[t]{2}{*}{ Factor } & \multirow[t]{2}{*}{ Full model } & \multicolumn{3}{|c|}{ Stratified by indication for antibiotics } \\
\hline & & $\begin{array}{l}\text { Antibiotics always } \\
\text { indicated }\end{array}$ & $\begin{array}{l}\text { Antibiotics may be } \\
\text { indicated }\end{array}$ & $\begin{array}{l}\text { Antibiotics never } \\
\text { indicated }\end{array}$ \\
\hline Number of encounters & 4474 & 762 & 1274 & 2438 \\
\hline Community ED (ref. urban ED) & $0.45(0.30,0.67)^{* * *}$ & $0.35(0.13,0.95)^{*}$ & $0.56(0.25,1.23)$ & $0.34(0.20,0.57)^{* * *}$ \\
\hline \multicolumn{5}{|l|}{ Provider type } \\
\hline Physician & Reference & Reference & Reference & Reference \\
\hline Physician assistant & $1.22(0.77,1.92)$ & $1.06(0.45,2.49)$ & $1.75(0.86,3.57)$ & $0.96(0.53,1.74)$ \\
\hline Resident & $0.72(0.46,1.13)$ & $1.41(0.42,4.73)$ & $0.78(0.35,1.76)$ & $0.54(0.30,0.98)^{*}$ \\
\hline \multicolumn{5}{|l|}{ Infection type } \\
\hline Acute respiratory infection & Reference & Reference & Reference & Reference \\
\hline Bronchitis & $4.55(3.40,6.08) * * *$ & - & $5.78(1.72,19.39) * *$ & $9.05(6.34,12.91) * * *$ \\
\hline Cough & $0.45(0.33,0.61) * * *$ & - & $1.00(1.00,1.00)^{* * *}$ & $0.92(0.65,1.29)$ \\
\hline Influenza & $0.25(0.16,0.38)^{* * *}$ & - & - & - \\
\hline Pneumonia & $\begin{array}{l}15.56(11.00 \\
22.00) * * *\end{array}$ & - & - & $0.54(0.34,0.86)^{* *}$ \\
\hline Streptococcal sore throat & $\begin{array}{l}69.79(25.62 \\
190.08)^{* * *}\end{array}$ & 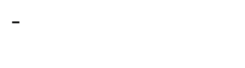 & $39.60(13.94,112.52)^{* * *}$ & 20 \\
\hline $\begin{array}{l}\text { Other diseases of the upper } \\
\text { respiratory tract }\end{array}$ & $2.09(1.62,2.70)^{* * *}$ & $1.75(0.60,5.06)$ & $3.33(1.92,5.78)^{* * *}$ & $0.75(0.46,1.21)$ \\
\hline Rapid treatment area & $0.43(0.32,0.56) * * *$ & $0.94(0.28,3.17)$ & $0.39(0.22,0.70)^{* *}$ & $0.43(0.30,0.63)^{* * *}$ \\
\hline Comorbidities ( 1 or more) & $1.27(0.97,1.65)$ & $2.07(0.47,9.16)$ & $1.00(0.54,1.85)$ & $1.38(0.99,1.92)$ \\
\hline Chief complaint respiratory infection & $1.31(1.12,1.54)^{* *}$ & $1.68(0.81,3.50)$ & $0.95(0.67,1.34)$ & $1.13(0.91,1.41)$ \\
\hline Patient age & $1.00(1.00,1.01)$ & $1.00(0.98,1.02)$ & $0.99(0.98,1.00)$ & $1.01(1.01,1.02)^{* * *}$ \\
\hline \multicolumn{5}{|l|}{ Patient race/ethnicity } \\
\hline Black, non-Hispanic & Reference & Reference & Reference & Reference \\
\hline White, non-Hispanic & $1.07(0.88,1.30)$ & $0.65(0.31,1.35)$ & $0.88(0.57,1.34)$ & $1.35(1.04,1.76) *$ \\
\hline Other race/ethnicity & $0.98(0.76,1.26)$ & $0.47(0.17,1.32)$ & $1.18(0.73,1.90)$ & $1.02(0.70,1.48)$ \\
\hline Female patient & $1.20(1.03,1.40) *$ & $1.76(0.93,3.31)$ & $1.31(0.95,1.79)$ & $1.23(0.99,1.52)$ \\
\hline Fever $\left(>100.4^{\circ} \mathrm{F}\right)$ & $1.84(1.35,2.51)^{* * *}$ & $1.11(0.43,2.84)$ & $2.89(1.47,5.67)^{* *}$ & $1.31(0.83,2.08)$ \\
\hline \multicolumn{5}{|l|}{ Time of day } \\
\hline $7 \mathrm{am}-3 \mathrm{pm}$ & Reference & Reference & Reference & Reference \\
\hline $3 \mathrm{pm}-11 \mathrm{pm}$ & $0.96(0.81,1.14)$ & $1.06(0.56,2.00)$ & $0.79(0.56,1.12)$ & $1.01(0.80,1.28)$ \\
\hline $11 \mathrm{pm}-7 \mathrm{am}$ & $0.62(0.49,0.79) * * *$ & $7.33(0.96,56.02)$ & $0.51(0.31,0.83)^{* *}$ & $0.60(0.43,0.83)^{* *}$ \\
\hline Long stay (>8 h) & $1.16(0.93,1.44)$ & $0.50(0.23,1.10)$ & $0.77(0.46,1.29)$ & $1.37(1.03,1.83)^{*}$ \\
\hline Influenza season & $0.96(0.82,1.13)$ & $0.64(0.34,1.22)$ & $1.33(0.95,1.85)$ & $0.99(0.79,1.23)$ \\
\hline Male provider & $0.89(0.62,1.29)$ & $0.95(0.45,2.02)$ & $0.77(0.41,1.43)$ & $1.04(0.65,1.68)$ \\
\hline \multicolumn{5}{|l|}{ Gists } \\
\hline Why not take a risk? & $1.28(1.06,1.54) *$ & $0.98(0.65,1.49)$ & $1.28(0.92,1.77)$ & $1.32(1.04,1.68)^{*}$ \\
\hline Antibiotics may be harmful & $0.81(0.67,0.98)^{*}$ & $1.40(0.88,2.22)$ & $0.52(0.38,0.73)^{* * *}$ & $0.94(0.74,1.21)$ \\
\hline
\end{tabular}

*Significant at the $5 \%$ level, ** significant at the $1 \%$ level, *** significant at the $0.1 \%$ level

decision — choosing between a patient being sick and a patient remaining sick but with the added burden of antibiotic side effects - should be more effective in influencing clinicians' prescribing decisions than current strategies. In this approach, the possibility of improvement with antibiotics is essentially nil, whereas the possibility of side effects is non-nil.

Previous interventions have often assumed that clinicians are poorly informed about treatment guidelines, and have focused on education to improve guideline adherence. ${ }^{18}$ However, guideline familiarity has been shown to be only modestly related to prescribing. ${ }^{35-37}$ Our results are consistent with an alternative mechanism for influencing prescribing behavior that directly addresses a predominant decision strategy, namely, to provide sick patients with at least the possibility of improvement, with negligible risks. Although social factors and simple routines may also drive prescribing, ${ }^{38-40}$ our results suggest that clinicians' decisions for an individual patient are based on their qualitative interpretations of the scientific knowledge regarding best practices. Interventions that combine gist-based education with behavioral nudges that regularly prompt clinicians to consider the potential harmful side effects of antibiotics may be most effective.
Our results, which demonstrated the predictive validity of categorical gists, included controls for patient characteristics. Similar to previous quantitative studies assessing antibiotic prescribing for URIs, we found that certain patient characteristics affected the likelihood of prescribing, such as patient gender, wait time, and comorbidities. ${ }^{6-8}$ The two EDs in this study serve different proportions of minority patients, and the difference in prescribing rate by department was highly correlated with race/ethnicity, which suggests that race may influence prescribing decisions, as described in prior studies. ${ }^{6,8}$ Our results are also consistent with previous research showing that certain types of clinicians, such as PAs and nurse practitioners, are more likely to prescribe, ${ }^{35}$ which may also be consistent with the hypothesized strategy of hedging when uncertain. ${ }^{41}$

\section{LIMITATIONS}

This study was conducted in only two acute care EDs. Acute care settings may differ from office-based settings, particularly with regard to patient satisfaction, as there is no long-term 
patient-clinician relationship, thus increasing clinician uncertainty. A larger, multisite trial across different outpatient settings is needed to provide more generalizable findings. Second, the study did not control for every factor that may drive prescribing, nor did it vary factors experimentally. Other studies suggest that factors such as time pressure, inertia, concerns about patient follow-up, decision fatigue, and unrealistic estimates of potential complications may influence prescribing. ${ }^{38 \text {, }}$ 42,43 We also did not include questions specific to antibiotic resistance. Despite the importance of this issue in public health, it has not been shown to be a motivating factor in ED prescribing, ${ }^{19}$ and thus was not a dominant gist tested. Third, use of ICD-9 diagnosis codes may introduce inaccuracies. For instance, a clinician treating a patient for a suspected bacterial infection may apply an alternative diagnostic code. Still, an outside firm makes diagnosis coding decisions in these EDs, so clinicians' discretion in coding was likely limited. In addition, patients' visits occurred prior to survey, removing the potential that the study biased prescribing decisions. Finally, antibiotic prescriptions were attributed to the final clinician on each record; however, multiple clinicians were involved in treatment in some cases.

\section{CONCLUSIONS}

Reducing inappropriate prescribing is crucial for preserving antibiotic effectiveness. Our results suggest that clinicians who prescribe at higher rates may perceive treatment as unlikely to be helpful, but nevertheless as offering the possibility of benefit, with negligible harm to the individual patient. Educational approaches that emphasize the non-negligible risks of unnecessary antibiotics should help to reshape clinicians' gist representations of prescribing options and, consequently, their decisions. The application of validated psychological theories to decision-making has shown promise in improving medical decision-making in other realms. ${ }^{41,44,45}$ FTT is a useful method for improving both our understanding of the drivers of decision-making and our ability to design validated educational tools to alter behavior.

Corresponding Author: Eili Y. Klein, PhD; Department of Emergency MedicineJohns Hopkins School of Medicine, 5801 Smith Avenue, Suite 3220, Office 265, Davis Building, Baltimore, MD 21209, USA (e-mail: eklein@jhu.edu).

\section{Compliance with Ethical Standards:}

Conflict of Interest: The authors declare that they do not have a conflict of interest.

\section{REFERENCES}

1. Gerber JS, Prasad PA, Russell Localio A, et al. Variation in Antibiotic Prescribing Across a Pediatric Primary Care Network. J Pediatr Infect Dis Soc. 2015;4(4):297-304. doi:10.1093/jpids/piu086.
2. Gerber JS, Newland JG, Coffin SE, et al. Variability in antibiotic use at children's hospitals. Pediatrics. 2010;126(6):1067-1073. doi:10.1542/ peds.2010-1275.

3. Fleming-Dutra KE, Hersh AL, Shapiro DJ, et al. Prevalence of Inappropriate Antibiotic Prescriptions Among US Ambulatory Care Visits, 2010-2011. JAMA. 2016;315(17):1864-1873. doi:10.1001/jama.2016. 4151.

4. Braykov NP, Morgan DJ, Schweizer ML, et al. Assessment of empirical antibiotic therapy optimisation in six hospitals: an observational cohort study. Lancet Infect Dis. 2014;14(12):1220-1227.

5. Fridkin SK, Baggs J, Fagan R, et al. Vital Signs: Improving Antibiotic Use Among Hospitalized Patients. Morb Mortal Wkly Rep. 2014;63:1-7.

6. Barlam TF, Morgan JR, Wetzler LM, Christiansen CL, Drainoni M-L. Antibiotics for Respiratory Tract Infections: A Comparison of Prescribing in an Outpatient Setting. Infect Control Hosp Epidemiol. 2015;36(02):153-159.

7. Xu KT, Roberts D, Sulapas I, Martinez O, Berk J, Baldwin J. Overprescribing of antibiotics and imaging in the management of uncomplicated URIs in emergency departments. BMC Emerg Med. 2013;13(1):7.

8. Gerber JS, Prasad PA, Localio AR, et al. Racial differences in antibiotic prescribing by primary care pediatricians. Pediatrics. 2013;131(4):677684. doi:10.1542/peds.2012-2500.

9. Bharathiraja R, Sridharan S, Chelliah LR, Suresh S, Senguttuvan M. Factors Affecting Antibiotic Prescribing Pattern in Pediatric Practice. Indian J Pediatr. 2005;72(10):877-879.

10. Livorsi D, Comer A, Matthias MS, Perencevich EN, Bair MJ. Factors influencing antibiotic-prescribing decisions among inpatient physicians: a qualitative investigation. Infect Control Hosp Epidemiol. 2015;36(09): 1065-1072.

11. Menéndez R, Torres A, Zalacain R, et al. Guidelines for the treatment of community-acquired pneumonia: predictors of adherence and outcome. Am J Respir Crit Care Med. 2005;172(6):757-762. doi:10.1164/rccm. 200411-1444OC.

12. Halm EA, Switzer GE, Mittman BS, Walsh MB, Chang C-CH, Fine MJ. What Factors Influence Physicians' Decisions to Switch from Intravenous to Oral Antibiotics for Community-acquired Pneumonia? J Gen Intern Med. 2001;16(9):599-605. doi:10.1046/j.1525-1497.2001.016009599.x.

13. Halm EA, Atlas SJ, Borowsky LH, et al. xUnderstanding physician adherence with a pneumonia practice guideline: effects of patient, system, and physician factors. Arch Intern Med. 2000;160(1):98-104.

14. Avorn J, Solomon DH. Cultural and economic factors that (mis)shape antibiotic use: the nonpharmacologic basis of therapeutics. Ann Intern Med. 2000; 133(2): 128-135.

15. Nyquist AC, Gonzales R, Steiner JF, Sande MA. Antibiotic prescribing for children with colds, upper respiratory tract infections, and bronchitis. JAMA. 1998;279(11):875-877.

16. Parker HM, Mattick $\mathbf{K}$. The determinants of antimicrobial prescribing among hospital doctors in England: a framework to inform tailored stewardship interventions. Br J Clin Pharmacol. 2016; 82(2):431-40. doi: $10.1111 /$ bcp. 12953.

17. Suda KJ, Hicks LA, Roberts RM, Hunkler RJ, Danziger LH. A national evaluation of antibiotic expenditures by healthcare setting in the United States, 2009. J Antimicrob Chemother. 2013; 68(3):715-8.

18. Md Rezal RS, Hassali MA, Alrasheedy AA, Saleem F, Md Yusof FA, Godman B. Physicians' knowledge, perceptions and behaviour towards antibiotic prescribing: a systematic review of the literature. Expert Rev Anti Infect Ther. 2015;13(5):665-680.

19. May L, Gudger G, Armstrong P, et al. Multisite exploration of clinical decision making for antibiotic use by emergency medicine providers using quantitative and qualitative methods. Infect Control Hosp Epidemiol. 2014;35(09):1114-1125.

20. Grijalva CG, Nuorti JP, Griffin MR. Antibiotic prescription rates for acute respiratory tract infections in US ambulatory settings. JAMA. 2009;302(7):758-766.

21. Wang EE, Einarson TR, Kellner JD, Conly JM. Antibiotic prescribing for Canadian preschool children: evidence of overprescribing for viral respiratory infections. Clin Infect Dis. 1999;29(1):155-160.

22. Dugas AF, Kirsch TD, Toerper M, et al. An Electronic Emergency Triage System to Improve Patient Distribution by Critical Outcomes. J Emerg Med. 2016;50(6):910-918. doi:10.1016/j.jemermed.2016.02.026.

23. Reyna VF, Adam MB. Fuzzy-trace theory, risk communication, and product labeling in sexually transmitted diseases. Risk Anal. 2003;23(2):325-342.

24. Reyna VF. A Theory of Medical Decision Making and Health: Fuzzy Trace Theory. Med Decis Making. 2008;28(6):850-865. doi:10.1177/ 0272989X08327066. 
25. Adam MB, Reyna VF. Coherence and correspondence criteria for rationality: experts' estimation of risks of sexually transmitted infections. J Behav Decis Mak. 2005;18(3): 169-186. doi:10.1002/bdm.493.

26. Reyna VF. Risk perception and communication in vaccination decisions: a fuzzy-trace theory approach. Vaccine. 2012;30(25):3790-3797. doi:10. 1016/j.vaccine.2011.11.070.

27. Broniatowski DA, Klein EY, Reyna VF. Germs are germs, and why not take a risk? Patients' expectations for prescribing antibiotics in an innercity emergency department. Med Decis Making. 2015;35(1):60-67. doi:10.1177/0272989X14553472.

28. Reyna VF, Chick CF, Corbin JC, Hsia AN. Developmental reversals in risky decision making intelligence agents show larger decision biases than college students. Psychol Sci. 2014; 25(1): 76-84..

29. DiStefano C, Zhu M, Mindrila D. Understanding and using factor scores: Considerations for the applied researcher. Pract Assess Res Eval. 2009; 14(20): 1-11.

30. Goldstein H, Browne W, Rasbash J. Multilevel modelling of medical data. Stat Med. 2002;21(21):3291-3315. doi:10.1002/sim.1264.

31. Mills B, Reyna VF, Estrada S. Explaining contradictory relations between risk perception and risk taking. Psychol Sci. 2008;19(5):429433.

32. Reyna VF, Estrada SM, DeMarinis JA, Myers RM, Stanisz JM, Mills BA. Neurobiological and memory models of risky decision making in adolescents versus young adults. J Exp Psychol Learn Mem Cogn. 2011;37(5):1125.

33. Reyna VF, Mills BA. Theoretically motivated interventions for reducing sexual risk taking in adolescence: A randomized controlled experiment applying fuzzy-trace theory. J Exp Psychol Gen. 2014;143(4):1627-48.

34. Mehrotra A, Linder JA. Tipping the Balance Toward Fewer Antibiotics. JAMA Intern Med. 2016;176(11):1649-50. doi:10.1001/jamainternmed. 2016.6254

35. Linder JA, Schnipper JL, Tsurikova R, Volk LA, Middleton B. Selfreported familiarity with acute respiratory infection guidelines and antibiotic prescribing in primary care. Int $\mathrm{J}$ Qual Health Care. 2010;22(6):469-475.
36. Finkelstein JA, Davis RL, Dowell SF, et al. Reducing antibiotic use in children: a randomized trial in 12 practices. Pediatrics. 2001;108(1): 1-7.

37. Gonzales R, Steiner JF, Lum A, Barrett PH. Decreasing antibiotic use in ambulatory practice: impact of a multidimensional intervention on the treatment of uncomplicated acute bronchitis in adults. JAMA. 1999;281(16): 1512-1519.

38. Linder JA, Doctor JN, Friedberg MW, et al. Time of day and the decision to prescribe antibiotics. JAMA Intern Med. 2014;174(12):2029-2031. doi:10.1001/jamainternmed.2014.5225.

39. Meeker D, Knight TK, Friedberg $\mathbf{M W}$, et al. Nudging guidelineconcordant antibiotic prescribing: a randomized clinical trial. JAMA Intern Med. 2014;174(3):425-431. doi:10.1001/jamainternmed.2013. 14191.

40. Meeker D, Linder JA, Fox CR, et al. Effect of Behavioral Interventions on Inappropriate Antibiotic Prescribing Among Primary Care Practices: A Randomized Clinical Trial. JAMA. 2016;315(6):562-570. doi:10.1001/ jama.2016.0275.

41. Reyna VF, Lloyd FJ. Physician decision making and cardiac risk: effects of knowledge, risk perception, risk tolerance, and fuzzy processing. J Exp Psychol Appl. 2006;12(3):179-195. doi:10.1037/1076-898X.12.3.179.

42. Teixeira Rodrigues A, Roque F, Falcão A, Figueiras A, Herdeiro MT. Understanding physician antibiotic prescribing behaviour: a systematic review of qualitative studies. Int J Antimicrob Agents. 2013;41(3):203212. doi:10.1016/j.ijantimicag.2012.09.003.

43. Tonkin-Crine S, Yardley L, Little P. Antibiotic prescribing for acute respiratory tract infections in primary care: a systematic review and meta-ethnography. J Antimicrob Chemother. 2011;66(10):2215-2223. doi:10.1093/jac/dkr279.

44. Fraenkel L, Peters E, Charpentier P, et al. Decision tool to improve the quality of care in rheumatoid arthritis. Arthritis Care Res. 2012;64(7):977-985.

45. Fraenkel L, Matzko CK, Webb DE, et al. Use of decision support for improved knowledge, values clarification, and informed choice in patients with rheumatoid arthritis. Arthritis Care Res. 2015;67(11):1496-1502. 